\title{
A comparative study for evaluating flurbiprofen effectiveness in postoperative pain
}

\author{
Ibrahim Dughaim Alreshidi ${ }^{1,2},{ }^{*}$ Yu Feng ${ }^{1}$ and Xie Han ${ }^{3}$ \\ ${ }^{1}$ Department of Clinical Pharmacy, China Pharmaceutical University, Nanjing P.R China \\ ${ }^{2}$ The Ministry of Higher Education, Saudi Arabia \\ ${ }^{3}$ Nanjing Drum Tower Hospital, Nanjing P.R, China
}

\begin{abstract}
Aim of the present study was to evaluate the effect of flurbiprofen, which is a member of non-steroidal anti-inflammatory drug group (NSAIDs), on postoperative pain treatment. From September 2013 to May 2014, total of 250 patients were surveyed to perform a systematic evaluation of postoperative pain by comparing flurbiprofen with two other kinds of NSAIDs (diclofenac and ketorolac) and isotonic saline in a double-blind, randomized, placebo-controlled study. Patients were randomized for treatment: 65 cases received flurbiprofen, 60 cases received diclofenac sodium, 60 cases received ketorolac and 65 cases received the placebo $(0.9 \%$ isotonic saline) (control group). After 24-hours of surgery patients treated with flurbiprofen, ketorolac, and diclofenac showed the lowermost PPI scores compared with those treated with $0.9 \%$ isotonic saline $(\mathrm{P}<0.05)$. Moreover, flurbiprofen-treated patients also had the lowest PRI $(R) T$ scores $(P<0.05)$. When the pain rating index was examined by subclass, a significantly lower PRI(R)S score was detected in the flurbiprofen group at 24 hours $(P<0.05)$. However, at the 96 -hour time point, no differences that were found in PPI and PRI[R] scores between the ketorolac, diclofenac, and flurbiprofen groups, whereas the control group was significantly less effective than the NSAID drugs. Flurbiprofen seemed to be the most effective NSAID for the treatment of pain after internal fixation of fracture, even though at 24 hours after surgery pain was at a maximum.
\end{abstract}

Key Words: Non-steroidal anti-inflammatory drugs, inflammatory pain, diclofenac, ketorolac, internal fixation of fracture, doubleblind randomized study.

\section{INTRODUCTION}

Internal Fixation of Fracture (IFF) is surgical procedure in orthopedics that includes the implementation of implants for the bone repairing, a concept of the mid-19th century and was made valid for usual treatment in the mid-20th century. An internal fixator may be made of stainless steel or titanium (Uhthoff et al., 2006). Bone pain is pain coming from the bone. It occurs as a consequence of an extensive range of pathological and/or physical conditions and may harshly impair the quality of life for patients who suffer from it (Luger et al., 2005). The periosteum of the bone has many nerve endings in it. When cracks or breaks occur in the bone those nerves send information to the brain that is professed as a deep aching pain. Any movements of these edges of the bone will stimulate those nerves, producing the pain to be worsened (Lian, 2007). In the case of bone fractures, surgical treatment is generally the most effective. Analgesics are recommended in combination with surgery to relieve pain of injured bone (Mercadante, 1997). Non-steroidal anti-inflammatory drugs (NSAIDs) are generally used for the symptomatic relief of postoperative pain and mild-to-moderate pain due to inflammation and tissue injury (Rossi, 2006). Flurbiprofen is a member of the phenylalkanoic acid derivative family of (NSAIDs) used to treat the pain and inflammation of arthritis (Brogden et al., 1979). Flurbiprofen is the analgesic drug most commonly used to alleviate pain, tenderness, swelling and stiffness caused by osteoarthritis

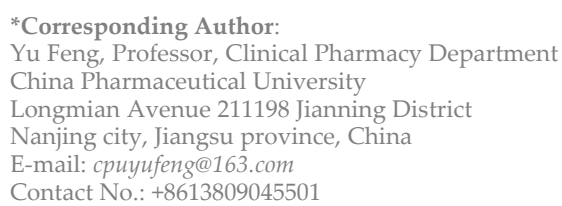

and rheumatoid arthritis (Brogden et al., 1979). Diclofenac is used to treat pain, inflammatory disorders, and dysmenorrhea (Bhaskar et al., 2010). Inflammatory disorders may include musculoskeletal complaints, especially arthritis, rheumatoid arthritis, polymyositis, dermatomyositis, osteoarthritis, dental pain, TMJ pain, spondylarthritis, ankylosing spondylitis, gout attacks. Ketorolac or ketorolac tromethamine is a (NSAID) belongs to the family of heterocyclic acetic acid derivatives, used as an analgesic. Ketorolac is indicated for short-term management of moderate to severe pain (Catapano et al., 1996). This study assessed the efficacy of three nonsteroidal anti-inflammatory drugs (NSAIDs) after Internal Fixation of Fracture (IFF) operation.

\section{PATIENTS AND METHODS}

This prospective, randomized, double-blind, placebocontrolled research was conducted at the Department of Orthopedic Surgery, Nanjing Drum tower hospital Nanjing, China. The research protocol was approved by the local department of ethics committee, and the research content was explained fully to each patient, and written informed consent was also obtained from all patients before the study began.

Using random number generation software, patients were randomly assigned to receive one kind of four topical agents: flurbiprofen $(50 \mathrm{mg}$ dissolved in $250 \mathrm{ml}$ physiological saline, intravenous drip, bid), diclofenac (50 $\mathrm{mg}$ dissolved in $250 \mathrm{ml}$ physiological saline, intravenous drip, bid), ketorolac (30mg dissolved in $250 \mathrm{ml}$ physiological saline, intravenous drip, bid), or vehicle $(0.9 \%$ isotonic saline, bid) (control group).

Quantitative pain analysis was done by using the McGill Pain Questionnaire. The McGill Pain 
Table 1: 250 patients of baseline demographic characteristics of the study population.*

\begin{tabular}{|c|c|c|c|c|c|}
\hline \multicolumn{2}{|c|}{ Characteristic } & \multirow{2}{*}{$\begin{array}{c}\text { flurbiprofen } \\
45\end{array}$} & \multirow{2}{*}{$\begin{array}{c}\text { diclofenac } \\
45\end{array}$} & \multirow{2}{*}{$\begin{array}{c}\text { ketorolac } \\
45\end{array}$} & \multirow{2}{*}{$\begin{array}{c}\text { isotonic saline } \\
45\end{array}$} \\
\hline Age, year & Mean & & & & \\
\hline & Range & $39-50$ & $38-51$ & $40-52$ & $41-50$ \\
\hline \multirow[t]{2}{*}{ Sex } & Male & 33 & 30 & 30 & 33 \\
\hline & Female & 32 & 30 & 30 & 32 \\
\hline \multirow[t]{2}{*}{ Height, cm } & Mean & 160 & 161 & 160 & 159 \\
\hline & Range & $155-180$ & $160-185$ & $158-182$ & $156-184$ \\
\hline \multirow[t]{2}{*}{ Body weight, kg } & Mean & 58 & 59 & 57 & 57 \\
\hline & Range & $46-75$ & $48-74$ & $45-70$ & $47-70$ \\
\hline
\end{tabular}

${ }^{*}$ No significant differences were found between different group, $\mathrm{p}<0.05$.

Table 2: Mean Present Pain Intensity (PPI) scores in the different treatment groups.

\begin{tabular}{|c|c|c|c|c|c|c|c|c|c|c|}
\hline \multirow{2}{*}{$\begin{array}{l}\text { Treatment group } \\
\text { for PPI score }\end{array}$} & \multicolumn{2}{|c|}{4 hours } & \multicolumn{2}{|c|}{24 hours } & \multicolumn{2}{|c|}{48 hours } & \multicolumn{2}{|c|}{72 hours } & \multicolumn{2}{|c|}{96 hours } \\
\hline & Mean & $\mathbf{P C}^{*}$ & Mean & PC $^{*}$ & Mean & $\mathbf{P C}^{*}$ & Mean & $\mathbf{P C}^{*}$ & Mean & $\mathrm{PC}^{*}$ \\
\hline Ketorolac (K) & 1.93 & $\mathrm{~K}-\mathrm{P}$ & 2.26 & $\mathrm{~K}-\mathrm{P}$ & 2.25 & $\mathrm{~K}-\mathrm{P}$ & 1.9 & $\mathrm{~K}-\mathrm{P}$ & 1.52 & $\mathrm{~K}-\mathrm{P}$ \\
\hline Diclofenac (D) & 2.26 & D-P & 2.29 & D-P & 2.21 & D-P & 2 & D-P & 1.65 & D-P \\
\hline Flurbiprofen $(\mathrm{F})$ & 1.66 & F-P,F-K,F-D & 1.86 & F-P,F-K,F-D & 1.72 & F-P,F-K,F-D & 1.34 & F-P,F-K,F-D & 1.26 & F-P,F-K,F-D \\
\hline Placebo $(\mathrm{P})$ & 2.77 & - & 3.08 & - & 2.92 & - & 2.62 & - & 2.48 & - \\
\hline $\mathrm{p}$ value & $<0.05$ & & $<0.05$ & & $<0.05$ & & $<0.05$ & & $<0.05$ & \\
\hline
\end{tabular}

Table 3: Total Pain Rating Index based on rank value (PRI[R]T) scores by treatment group (PRI[R]T score range: 0 to 100).

\begin{tabular}{|c|c|c|c|c|c|c|c|c|c|c|}
\hline \multirow{2}{*}{$\begin{array}{l}\text { Treatment group } \\
\text { for PRI(R)T score }\end{array}$} & \multicolumn{2}{|c|}{4 hours } & \multicolumn{2}{|c|}{24 hours } & \multicolumn{2}{|c|}{48 hours } & \multicolumn{2}{|c|}{72 hours } & \multicolumn{2}{|c|}{96 hours } \\
\hline & Mean & $\mathrm{PC}^{*}$ & Mean & PC $^{*}$ & Mean & $\mathrm{PC}^{*}$ & Mean & $\mathrm{PC}^{*}$ & Mean & PC $^{*}$ \\
\hline Ketorolac(K) & 13.24 & K-P & 13.09 & $\mathrm{~K}-\mathrm{P}$ & 12.87 & $\mathrm{~K}-\mathrm{P}$ & 12.96 & $\mathrm{~K}-\mathrm{P}$ & 1.52 & $\mathrm{~K}-\mathrm{P}$ \\
\hline Diclofenac(D) & 14.88 & $\mathrm{D}-\mathrm{P}$ & 15.77 & D-P & 15.24 & D-P & 13.11 & D-P & 1.65 & D-P \\
\hline Flurbiprofen(F) & 12.92 & F-P & 12.09 & F-P,F-K,F-D & 10.39 & F-P,F-K,F-D & 8.00 & F-P,F-K,F-D & 1.26 & F-P,F-K,F-D \\
\hline Placebo(P) & 22.40 & - & 24.03 & - & 20.60 & - & 18.01 & - & 2.48 & - \\
\hline $\mathrm{p}$ value & $<0.05$ & & $<0.05$ & & $<0.05$ & & $<0.05$ & & $<0.05$ & \\
\hline
\end{tabular}

*PC, pairwise comparisons; K-P, D-P, etc., indicate which two drugs were being compared.

Questionnaire involves the measurement of three major factors: (1) the pain rating index, (2) pain intensity (3) and the number of words chosen. Response to treatment was evaluated by patients' rating of pain intensity using the Present Pain Intensity (PPI). Pain intensity was recorded at four hours postoperatively and then once daily on days 1 to 4 . Patients described the pain using a six-category descriptor scale, circling the term that best reflected the amount of pain and rating the pain as follows: $0=$ none, 1 $=$ mild, 2 = discomforting, $3=$ distressing, $4=$ horrible, $5=$ severe, and $6=$ excruciating. Pain rating index based on the rank values of the words (PRIR]) consisted of 4 subscales-sensory (S), affective (A), evaluative (E), and miscellaneous $(\mathrm{M})$-as well as a total score $(\mathrm{T})$. In this scoring system of the PRI(R), the word in each subclass suggesting the pain intensity, 1 value point out least pain, the next word is given a value of 2 , etc. The values of the words chosen by a patient are then added up to obtain a score for each category, and a total score for all categories.

Patients were monitored and asked if they had experienced any adverse events after surgery of 24 hours by a blinded investigator. The same investigator interviewed each patient and recorded symptoms and signs associated with general malaise or local discomfort at the site of injection. When an adverse event was reported, the patient was asked to rate its severity, and to approximate its duration.

\section{Statistical analysis}

All statistical results were expressed as mean \pm standard deviation $\overline{(\bar{x}} \pm s)$ for each group. SPSS $\AA 19.0$ software was used to undertake statistical analysis. Between-group differences were used to analyze by Fisher's exact test in categorical variables. Between-group differences of continuous variable were used to assess by Student's ttest. A p-value less than 0.05 was considered to be statistically significant.

\section{RESULTS}

A total of 250 patients between January 2013 and May 2014 were selected in our hospital that needed surgery due to bone fracture. Thus, there were 126 men and 124 women; age from 38 to 52 years, (mean age 45 years), height 155 to $185 \mathrm{~cm}$ (mean height $160 \mathrm{~cm}$ ); body weight 45 to75 $\mathrm{kg}$ (mean body weight $58 \mathrm{~kg}$ ). The flurbiprofen group was comprised of 65 patients, the diclofenac group was comprised of 60 patients, the ketorolac group was comprised of 60 patients, and the control group was comprised of 65 patients. The preoperative statistics showed patients' demographic characteristics were not statistically significance between the different four treatment groups (table 1 ).

Mean PPI scores were recorded in table 2. From the table we have cognized that between-group differences were significant $(\mathrm{P}<0.05)$. Comparing with the placebo group, mean PPI scores were significantly lower for all NSAID groups at every time points than control group $(\mathrm{P}<0.05)$. Further analysis of PPI scores at four hours after surgery revealed no significant differences between ketorolac, diclofenac, and flurbiprofen in the self-reported scores. In contrast, the flurbiprofen group reported mean PPI scores significantly $(\mathrm{P}<0.05)$ lower than did the diclofenac and ketorolac groups. No significant differences were found between NSAID groups when PRI(R)T 
scores were compared at the four hour time point; the control group was significantly less effective in pain relief than any of the three kinds of NSAIDs $(\mathrm{P}<0.001)$.

Most patients reported the highest mean PPI and PRI(R)T scores at the 24-hour time point. At this time, the lowest PPI scores were observed in the flurbiprofen, and diclofenac groups, all of which had significantly lower scores than the ketorolac, or placebo groups $(\mathrm{P}<0.05)$. Flurbiprofen-treated patients reported significantly $(\mathrm{P}<0.05)$ lower PRI $(\mathrm{R}) \mathrm{T}$ and $\mathrm{PRI}(\mathrm{R}) \mathrm{S}$ scores after 24 hours compared with any of the other treatment groups, as shown in Tables III. At the same time point, PRI(R)A scores did not differ significantly between the flurbiprofen, diclofenac, and ketorolac groups, whereas the placebo and indomethacin groups reported significantly $(\mathrm{P}<0.05)$ higher scores than all other groups. PRI(R)E and PRI(R)M were significantly higher in the placebo group compared with each of the NSAIDs ( $\mathrm{P}<0.05$ for each comparison). However, there were no significant differences in pairwise comparisons between NSAIDs at any time. At the ninetysix hour time points, no differences were found in PPI and PRI[R] scores between the ketorolac, diclofenac, and flurbiprofen groups, whereas the control group was significantly less effective than the NSAID drugs.

All the treatment groups were well tolerated for different NSAIDs. The only adverse event reported during the treatment period was transient fever after intravenous drip. Subjective symptoms of transient fever did not appear to affect flurbiprofen and isotonic saline administration groups. A subset of patients $(15 / 120,12.5 \%)$ who received ketorolac $(9 / 120,7.5 \%)$ and diclofenac $(6 / 120,5 \%)$ reported some fever compared with those in the other treatment groups.

\section{DISCUSSION}

This study compared the analgesic effect and drug safety of flurbiprofen with other those of NSAID, such as Ketorolac and Diclofenac, in the treatment of pain resulting from Internal Fixation of Fracture. In the present study, all NSAID drug groups had significantly lower PPI scores and PRI(R)T scores than the isotonic saline group at every time points. From the data, flurbiprofen seemed to be the most effective NSAID in relieving pain after surgery.

Comparing the PPI scores or PRI(R)T scores of the different NSAIDs groups showed different results at the time points of four hours, twenty-four hours, forty-eight hours, seventy-two hours and ninety-six hours. Selfreported results of patients' pain showed characteristically high peaks at twenty-four hours after surgery. Based on the analysis of the PPI and PRI(R)T scores reveals that the PPI scores at four hour time point were the lowest for the flurbiprofen, ketorolac, and diclofenac groups. In addition, there was no statistically significant difference between the different groups. However, the mean PPI score was significantly lower in the flurbiprofen group than in ketorolac or diclofenac groups, while the PRI(R)T scores were significantly lower in the ketorolac group than in the flurbiprofen group, diclofenac group or placebo groups. The fact is that PPI and PRI(R)T scores did not show the same differences between different NSAID groups. This may be because the PPI score is based on the single choice of a number-word. This designation is clearly subjective and lacks enough degrees of expression whereas PRI(R) scores involve multiple choices and are less reliable. Moreover, PPI scores fluctuate considerably according to psychological factors, whereas PRI(R) scores are less influenced by variables other than the sensory dimensions of pain. The same observations also could be applied to the visual analog rating scale or to other subjective, number-word-based scales.

It should be noted that at 24 hours after surgery, PRI(R)S scores significantly decreased in the flurbiprofen group compared with all other treatment groups $(\mathrm{P}<0.05$, whereas PRI(R)A scores in the flurbiprofen groups, ketorolac groups, and diclofenac groups did not show significantly statistical differences. This finding confirmed that flurbiprofen was significantly more effective analgesic drug compared with the other NSAIDs treatment groups and that this result was not biased by the affective component of pain.

At forty-eight hours and seventy-two hours after surgery, all PPI and PRI(R)T scores were reduced compared with the previous time point. Moreover, patients who received flurbiprofen still reported significantly lower PPI and PRI(R)T scores compared with the other groups. At the ninety-six hour time point after surgery, however, no differences that were found in PPI and PRI[R] scores between the ketorolac, diclofenac, and flurbiprofen groups, whereas the control group was significantly less effective than the NSAID drugs.

Subjective symptoms, except for transient fever which was more likely and would be considered an early complication or adverse effects of NSAIDs administration, did not significantly difference between groups. However, only a small proportion of ketorolac-treated or diclofenactreated patients reported transient fever. The reason for this has been well established, in that the toxicity of protection mechanisms, the transient fever associated with ketorolac-treated or diclofenac-treated patients may have been related to the presence of these protection mechanisms. However, further investigation is needed to evaluate this point.

\section{CONCLUSION}

In conclusions, flurbiprofen seemed to be the most effective NSAID drug for the treatment of post-operative pain after internal fixation of fracture surgery, even though pain was at a maximum degree at 24 hours after surgery.

\section{REFERENCES}

Bhaskar, H., Kapoor. P. and Ragini. (2010). Comparison of transdermal diclofenac patch with oral diclofenac as an analgesic modality following multiple premolar extractions in orthodontic patients: A cross over efficacy trial. Contemp Clin Dent. 1(3): 158-163. [DOI] PMid:22114407

Brogden, R. N., Heel, R. C., Speight, T. M., Avery, G. S. (1979). Flurbiprofen: a review of its pharmacological properties and therapeutic use in rheumatic diseases, Drugs, 18(6): 417-438. [DOI]

Catapano, M.S. (1996). The analgesic efficacy of ketorolac for acute pain, The Journal of Emergency Medicine, 14(1): 67-75. [DOI]

Lian, G. (2007). [Link]

Luger, N., Mach, D., Sevcik, M., Mantyh, P. (2005). Bone cancer pain: From mechanism to model to therapy. Journal of Pain and Symptom Management. 29(5): 32-46. [DOI] PMid:15907645

Mercadante, S (1997). Malignant bone pain: Pathophysiology and treatment, Pain, 69(1-2): 1-18. [DOI]

Rossi, S. (2006). Australian Medicines Handbook, Adelaide: Australian Medicines Handbook Pty Ltd. ISBN 0-9757919-2-3.

Uhthoff, H.K., Poitras, P., Backman, D.S. (2006). Internal plate fixation of fractures: short history and recent developments. J Orthop Sci., 11(2): 118-126. [DOI] 